\title{
On a Durrmeyer-type modification of the Exponential sampling series
}

\author{
Carlo Bardaro $^{1}$ (D) . Ilaria Mantellini ${ }^{1}$
}

Received: 5 August 2020 / Accepted: 3 September 2020 / Published online: 17 September 2020

(c) The Author(s) 2020

\begin{abstract}
In this paper we introduce the exponential sampling Durrmeyer series. We discuss pointwise and uniform convergence properties and an asymptotic formula of Voronovskaja type. Quantitative results are given, using the usual modulus of continuity for uniformly continuous functions. Some examples are also described.
\end{abstract}

Keywords Exponential sampling Durrmeyer series $\cdot$ Mellin derivatives $\cdot$ Moments Voronovskaja formula $\cdot$ Modulus of continuity

Mathematics Subject Classification $42 \mathrm{C} 15 \cdot 46 \mathrm{E} 22 \cdot 94 \mathrm{~A} 20$

\section{Introduction}

The theory of the exponential sampling series of (real or complex)-valued functions $f$ defined over the positive real axis, is a powerful tool for investigating certain phenomena in optical physics, as for example, light scattering, Fraunhofer diffraction etc, (see e.g. [16, 19, 26, 29]). From a mathematical point of view these series were rigorously studied in [18], (see also [4]). The suitable frame for studying these operators is the Mellin analysis, in particular the Mellin transform theory (see [17, 28]). Indeed, the exponential sampling operator represents the counterpart of the classical Shannon sampling series of Fourier analysis (see [27]) in Mellin setting. Now the samples are not equally spaced, but exponentially spaced over the positive real axis, and the classical "sinc" kernel is now replaced by a composition of the sinc-function with the logarithm. The exponential sampling series

Carlo Bardaro and Ilaria Mantellini have been partially supported by the "Gruppo Nazionale per l'Analisi Matematica e Applicazioni (GNAMPA)" of the "Instituto di Alta Matematica (INDAM)" as well as by the project "Ricerca di Base 2019 of University of Perugia (title: Integrazione, Approssimazione Analisi non Lineare e loro Applicazioni)".

Carlo Bardaro

carlo.bardaro@unipg.it

Ilaria Mantellini

ilaria.mantellini@unipg.it

1 Department of Mathematics and Computer Sciences, University of Perugia, via Vanvitelli 1, 06123 Perugia, Italy 
enables one to reconstruct functions (signals) which are Mellin-bandlimited. Indeed, the study of the structure of the class of Mellin-bandlimited functions is a deep topic of Mellin analysis. It was studied in [5, 6], in terms of Mellin-Bernstein spaces.

As for the Shannon sampling series, later on a generalized version of the exponential sampling series was introduced in [9] (see also [3, 15]), in which the "sinc-log" kernel is replaced by a function $\varphi$ defined on $\mathbb{R}^{+}$satisfying suitable assumptions. This is very important in order to obtain reconstructions of functions not necessarily Mellin band-limited, and to develop a "prediction" theory as a counterpart of the theory developed in [8] for the generalized sampling series of Fourier analysis.

In [7] the classical generalized sampling series of Fourier analysis was modified by replacing the sample values of the function $f$ with its mean-value in small intervals, so defining the Kantorovich sampling series. This represents a wide field of investigations, due to its practical applications in various sectors of applied sciences (see e.g. [1, 2, 20-25] and references therein).

In [13] (see also [10] and [14] for a multivariate version), a further extension of the generalized Kantorovich sampling series was introduced, by replacing the mean-values of the function $f$ by "approximating values" of $f$ defined through general convolution operator, obtaining the so-called Durrmeyer generalized sampling type series. Note that a general approach to sampling series in functional spaces was developed in [31].

In the present paper we present an analogous generalization for the exponential sampling series, using Mellin convolution operators. The present paper represents a first step in the construction of the approximation theory for semi-discrete operators in Mellin setting: here we obtain pointwise and uniform convergence theorems, giving also a quantitative version in terms of the so-called log-modulus of continuity (see Sect. 5), and an asymptotic formula of Voronovskaja type under certain local regularity assumptions on the function $f$. The last section is devoted to some examples, involving the central B-splines (see $[8,15$, 30]) and the Mellin-Jackson kernel (see [12]). Many other examples can be constructed.

\section{Preliminaries}

Let us denote by $\mathbb{N}, \mathbb{N}_{0}$ and $\mathbb{Z}$ the sets of positive integers, nonnegative integers and integers respectively. Moreover by $\mathbb{R}$ and $\mathbb{R}^{+}$we denote the sets of all real and positive real numbers respectively.

In what follows, for simplicity, we will assume that the functions $f$ defined on $\mathbb{R}^{+}$take their values in $\mathbb{R}$, but the results remain valid also for complex-valued functions.

Let $L^{\infty}\left(\mathbb{R}^{+}\right)$be the space of all the essentially bounded functions $f: \mathbb{R}^{+} \rightarrow \mathbb{R}$ endowed with the usual norm $\|f\|_{\infty}$.

Moreover we will denote by $C=C\left(\mathbb{R}^{+}\right)$the space of all the continuous functions $f: \mathbb{R}^{+} \rightarrow \mathbb{R}$ and by $C^{0}=C^{0}\left(\mathbb{R}^{+}\right)$the space of all the uniformly continuous and bounded functions on $\mathbb{R}^{+}$.

We say that a function $f \in C\left(\mathbb{R}^{+}\right)$is "log-uniformly continuous" on $\mathbb{R}^{+}$, if for any $\varepsilon>0$ there exists $\delta>0$ such that

$$
|f(u)-f(v)|<\varepsilon \text { whenever }|\log u-\log v| \leq \delta \quad\left(u, v \in \mathbb{R}^{+}\right) .
$$

We denote by $\mathcal{C}\left(\mathbb{R}^{+}\right)$the space containing the log-uniformly continuous and bounded functions $f: \mathbb{R}^{+} \rightarrow \mathbb{R}$. Note that in general a log-uniformly continuous function is not 
necessarily uniformly continuous and conversely. Obviously the two notions are equivalent on compact intervals in $\mathbb{R}^{+}$.

For a function $f \in C^{0}$ and $r \in \mathbb{N}$ we will say that $f$ belongs to $C^{r}$ locally at a point $x \in \mathbb{R}^{+}$if there is a neighbourhood $U$ of $x$ such that $f$ is $(r-1)$-fold continuously differentiable in $U$ and $f^{(r)}(x)$ exists.

The pointwise Mellin differential operator $\Theta$, or the pointwise Mellin derivative $\Theta f$ of a function $f: \mathbb{R}^{+} \rightarrow \mathbb{R}$, is defined by (see [17])

$$
\Theta f(x):=x f^{\prime}(x), \quad x \in \mathbb{R}^{+}
$$

provided $f^{\prime}(x)$ exists on $\mathbb{R}^{+}$. The Mellin differential operator of order $r \in \mathbb{N}$ is defined iteratively by

$$
\Theta^{1}:=\Theta, \quad \Theta^{r}:=\Theta\left(\Theta^{r-1}\right) .
$$

For convenience set $\Theta^{0}:=I, I$ denoting the identity. For instance, the first three Mellin derivatives are given by:

$$
\begin{aligned}
\Theta f(x) & =x f^{\prime}(x), \\
\Theta^{2} f(x) & =x^{2} f^{\prime \prime}(x)+x f^{\prime}(x), \\
\Theta^{3} f(x) & =x^{3} f^{\prime \prime \prime}(x)+3 x^{2} f^{\prime \prime}(x)+x f^{\prime}(x) .
\end{aligned}
$$

In general, we have (see [17])

$$
\Theta^{r} f(x)=\sum_{k=0}^{r} S(r, k) x^{k} f^{(k)}(x),
$$

where $S(r, k), 0 \leq k \leq r$, denote the (classical) Stirling numbers of second kind. We have the following Taylor formula with Mellin derivatives (see [11, 28]).

Proposition 1 For any $f \in C^{0}\left(\mathbb{R}^{+}\right)$belonging to $C^{r}$ locally at a point $x \in \mathbb{R}^{+}$we have

$$
f(t x)=f(x)+(\Theta f)(x) \log t+\frac{\left(\Theta^{2} f\right)(x)}{2 !} \log ^{2} t+\cdots+\frac{\left(\Theta^{r} f\right)(x)}{r !} \log ^{r} t+h(t) \log ^{r} t,
$$

where $h: \mathbb{R}^{+} \rightarrow \mathbb{R}$ is a bounded function such that $h(t) \rightarrow 0$ for $t \rightarrow 1$.

Remark 1 The boundedness of the function $h$ in the remainder of the Taylor formula with Mellin derivatives comes from the boundedness of the function $f$. However, the same holds for functions $f \in C\left(\mathbb{R}^{+}\right)$which have a growth of type

$$
|f(x)| \leq a+b|\log x|^{r},
$$

for positive constants $a, b$ and $x \in \mathbb{R}^{+}$. Indeed, employing the limit relation at the point 1 the function $h$ is obviously bounded in an interval containing 1, while using the growth condition on $f$ and expressing $h$ in terms of the Taylor formula, one can see easily the boundedness of $h$ in the complement of the interval. 


\section{Exponential sampling Durrmeyer operator}

Let $\varphi: \mathbb{R}^{+} \rightarrow \mathbb{R}$ be a continuous function such that the following assumptions are satisfied

( $\varphi .1) \quad$ for every $u \in \mathbb{R}^{+}, \sum_{k=-\infty}^{\infty} \varphi\left(e^{-k} u\right)=1$;

$(\varphi .2) \quad$ we have that

$$
M_{0}(\varphi)=\sup _{u \in \mathbb{R}^{+}} \sum_{k=-\infty}^{\infty}\left|\varphi\left(e^{-k} u\right)\right|<+\infty
$$

( $\varphi .3)$ for some $v \in \mathbb{N}$,

$$
\lim _{r \rightarrow+\infty} \sum_{\left|\log \left(e^{-k} x^{n}\right)\right|>r}\left|\varphi\left(e^{-k} u\right)\right||k-\log u|^{v}=0 .
$$

uniformly with respect to $u \in \mathbb{R}^{+}$;

we denote by $\Phi$ the class of all functions $\varphi$ satisfying the above assumptions.

Let $\psi: \mathbb{R}^{+} \rightarrow \mathbb{R}$ be a function with the following conditions

$(\psi .1)$

$$
\int_{0}^{\infty} \psi(t) \frac{d t}{t}=1
$$

$$
\widetilde{M}_{0}(\psi):=\int_{0}^{\infty}|\psi(t)| \frac{d t}{t}<+\infty .
$$

and let us denote by $\Psi$ the class of all functions $\psi$ satisfying the above assumptions.

Let $\nu \in \mathbb{N}_{0}$. For $x \in \mathbb{R}^{+}$we define the algebraic moments of order $\nu$ of $\varphi \in \Phi$ and $\psi \in \Psi$ as

$$
m_{\nu}(\varphi, x):=\sum_{k=-\infty}^{\infty} \varphi\left(e^{-k} x\right) \log ^{\nu}\left(e^{k} x^{-1}\right)=\sum_{k=-\infty}^{\infty} \varphi\left(e^{-k} x\right)(k-\log (x))^{\nu}
$$

and

$$
\tilde{m}_{\nu}(\psi):=\int_{0}^{\infty} \psi(t) \log ^{\nu} t \frac{d t}{t} .
$$

The absolute moments of order $v$ of $\varphi \in \Phi$ and $\psi \in \Psi$ are defined as

$$
M_{\nu}(\varphi, x):=\sum_{k=-\infty}^{\infty}\left|\varphi\left(e^{-k} x\right)\right|\left|\log ^{v}\left(e^{k} x^{-1}\right)\right|=\sum_{k=-\infty}^{\infty}\left|\varphi\left(e^{-k} x\right)\right||k-\log (x)|^{\nu} .
$$

We will set $M_{\nu}(\varphi):=\sup _{x \in \mathbb{R}^{+}} \sum_{k=-\infty}^{\infty}\left|\varphi\left(e^{-k} x\right)\right|\left|\log ^{v}\left(e^{k} x^{-1}\right)\right|$ and

$$
\tilde{M}_{\nu}(\psi):=\int_{0}^{\infty}|\log t|^{v}|\psi(t)| \frac{d t}{t} .
$$

Remark 2 Note that, for $\varphi \in \Phi$, if $\mu, v \in \mathbb{N}$ with $\mu<v$, then $M_{\nu}(\varphi)<\infty$ implies $M_{\mu}(\varphi)<\infty$. Indeed for $\mu<v$ we have 


$$
\begin{aligned}
\sum_{k=-\infty}^{\infty}\left|\varphi\left(e^{-k} x\right)\right||k-\log x|^{\mu} & =\left(\sum_{|k-\log x|<1}+\sum_{|k-\log x| \geq 1}\right)\left|\varphi\left(e^{-k} x\right)\right||k-\log x|^{\mu} \\
& \leq M_{0}(\varphi)+\sum_{|k-\log x| \geq 1}\left|\varphi\left(e^{-k} x\right)\right||k-\log x|^{\nu} \\
& \leq M_{0}(\varphi)+M_{\nu}(\varphi) .
\end{aligned}
$$

The same for the absolute moments of $\psi \in \Psi$. Indeed we may write for $\mu<v$

$$
\begin{aligned}
\int_{0}^{\infty}|\psi(t)||\log t|^{\mu} \frac{d t}{t} & =\int_{|\log t|<1}|\psi(t)||\log t|^{\mu} \frac{d t}{t}+\int_{|\log t| \geq 1}|\psi(t)||\log t|^{\mu} \frac{d t}{t} \\
& \leq \widetilde{M}_{0}(\psi)+\int_{|\log t| \geq 1}|\psi(t)||\log t|^{\nu} \frac{d t}{t} \\
& \leq \widetilde{M}_{0}(\psi)+\widetilde{M}_{\nu}(\psi) .
\end{aligned}
$$

Let $\varphi \in \Phi$ and $\psi \in \Psi$. For any $n \in \mathbb{N}$, and $f: \mathbb{R}^{+} \rightarrow \mathbb{R}$, we define the exponential sampling Durrmeyer series as

$$
\left(S_{n}^{\varphi, \psi} f\right)(x):=\sum_{k=-\infty}^{\infty} \varphi\left(e^{-k} x^{n}\right) n \int_{0}^{\infty} \psi\left(e^{-k} u^{n}\right) f(u) \frac{d u}{u}
$$

for $x \in \mathbb{R}^{+}$and for any function $f \in \operatorname{dom} S_{n}^{\varphi, \psi}$, being dom $S_{n}^{\varphi, \psi}$ the set of all functions $f$ for which the series is absolutely convergent at every $x$. Using the conditions of the classes $\Phi$ and $\Psi$, it is easy to see that the above operator is well defined as an absolutely convergent series, for any function $f \in L^{\infty}\left(\mathbb{R}^{+}\right)$. In particular $C^{0}\left(\mathbb{R}^{+}\right) \subset \operatorname{dom} S_{n}^{\varphi, \psi}$, for any $n \in \mathbb{N}$. Indeed, putting $t=e^{-k} u^{n}$

$$
\sum_{k=-\infty}^{\infty}\left|\varphi\left(e^{-k} x^{n}\right)\right| n \int_{0}^{\infty}\left|\psi\left(e^{-k} u^{n}\right)\right||f(u)| \frac{d u}{u} \leq\|f\|_{\infty} \widetilde{M}_{0}(\psi) M_{0}(\varphi) .
$$

We can determine larger subspaces of the domain of $S_{n}^{\varphi, \psi}$. We admit functions which grow like a power of the logarithm. We have the following (see also [15])

Proposition 2 Let $\varphi \in \Phi$ and $\psi \in \Psi$. Suppose that $M_{r}(\varphi)<\infty$ and $\widetilde{M}_{r}(\psi)<\infty$ for some $r \in \mathbb{N}$. If $f: \mathbb{R}^{+} \rightarrow \mathbb{R}$ and $|f(x)| \leq a+b|\log x|^{r}$ for some $a, b \in \mathbb{R}^{+}$and all $x \in \mathbb{R}^{+}$, then $f$ belongs to the domain of $S_{n}^{\varphi, \psi}$.

Proof We prove the proposition considering $r=2$ because the general case follows in an analogous way. So assume that $|f(x)| \leq a+b|\log x|^{2}$ with $a, b \in \mathbb{R}^{+}$.

$$
\begin{gathered}
\left|\left(S_{n}^{\varphi, \psi} f\right)(x)\right| \leq \sum_{k=-\infty}^{\infty}\left|\varphi\left(e^{-k} x^{n}\right)\right| n a \int_{0}^{\infty}\left|\psi\left(e^{-k} u^{n}\right)\right| \frac{d u}{u} \\
+\sum_{k=-\infty}^{\infty}\left|\varphi\left(e^{-k} x^{n}\right)\right| n b \int_{0}^{\infty}\left|\psi\left(e^{-k} u^{n}\right)\right||\log u|^{2} \frac{d u}{u} .
\end{gathered}
$$

Then, using the change of variable $\left(t e^{k}\right)^{1 / n}=u$ in the above integral, we obtain 


$$
\begin{aligned}
& \left|\left(S_{n}^{\varphi, \psi} f\right)(x)\right| \leq a \sum_{k=-\infty}^{\infty}\left|\varphi\left(e^{-k} x^{n}\right)\right| \int_{0}^{\infty}|\psi(t)| \frac{d t}{t} \\
& \quad+b \sum_{k=-\infty}^{\infty}\left|\varphi\left(e^{-k} x^{n}\right)\right| \int_{0}^{\infty}|\psi(t)|\left|\log \left(t e^{k}\right)^{1 / n}\right|^{2} \frac{d t}{t} \\
& \leq a M_{0}(\varphi) \tilde{M}_{0}(\psi)+\frac{b}{n^{2}} \sum_{k=-\infty}^{\infty}\left|\varphi\left(e^{-k} x^{n}\right)\right| \int_{0}^{\infty}|\psi(t)|(|\log t|+|k|)^{2} \frac{d t}{t} \\
& \leq a M_{0}(\varphi) \tilde{M}_{0}(\psi)+\frac{b}{n^{2}} \sum_{k=-\infty}^{\infty}\left|\varphi\left(e^{-k} x^{n}\right)\right| \int_{0}^{\infty}|\psi(t)|\left(|\log t|^{2}+2|k||\log t|+k^{2}\right) \frac{d t}{t} \\
& \leq a M_{0}(\varphi) \tilde{M}_{0}(\psi)+\frac{b}{n^{2}} M_{0}(\varphi) \tilde{M}_{2}(\psi)+\frac{2 b}{n^{2}} \sum_{k=-\infty}^{\infty}\left|\varphi\left(e^{-k} x^{n}\right)\right||k| \int_{0}^{\infty}|\psi(t)||\log t| \frac{d t}{t} \\
& \quad+\frac{b}{n^{2}} \sum_{k=-\infty}^{\infty}\left|\varphi\left(e^{-k} x^{n}\right)\right| k^{2} \int_{0}^{\infty}|\psi(t)| \frac{d t}{t} \\
& \leq a M_{0}(\varphi) \tilde{M}_{0}(\psi)+\frac{b}{n^{2}} M_{0}(\varphi) \tilde{M}_{2}(\psi)+\frac{2 b}{n^{2}} \tilde{M}_{1}(\psi) \sum_{k=-\infty}^{\infty}\left|\varphi\left(e^{-k} x^{n}\right)\right||k| \\
& \quad+\frac{b}{n^{2}} \tilde{M}_{0}(\psi) \sum_{k=-\infty}^{\infty}\left|\varphi\left(e^{-k} x^{n}\right)\right| k^{2} .
\end{aligned}
$$

Taking in to account that $k^{2} \leq 2\left(\left(k-\log x^{n}\right)^{2}+\log ^{2} x^{n}\right)$ and $|k| \leq\left|k-\log x^{n}\right|+\left|\log x^{n}\right|$ we obtain

$$
\begin{aligned}
& \left|\left(S_{n}^{\varphi, \psi} f\right)(x)\right| \leq a M_{0}(\varphi) \tilde{M}_{0}(\psi)+\frac{b}{n^{2}} M_{0}(\varphi) \tilde{M}_{2}(\psi)+\frac{2 b}{n^{2}} \widetilde{M}_{1}(\psi) M_{1}(\varphi) \\
& \quad+\frac{2 b}{n^{2}} \widetilde{M}_{1}(\psi) M_{0}(\varphi)\left|\log x^{n}\right|+\frac{b}{n^{2}} \widetilde{M}_{0}(\psi) M_{2}(\varphi)+\frac{b}{n^{2}} \widetilde{M}_{0}(\psi) M_{0}(\varphi) \log ^{2} x^{n} .
\end{aligned}
$$

The general case is obtained by $(|\log t|+|k|)^{r} \leq 2^{r-1}\left(|\log t|^{r}+|k|^{r}\right)$.

\section{Pointwise and uniform convergence}

In this section we state a pointwise convergence theorem at continuity points of the function $f$. Then we obtain as a corollary, a uniform convergence theorem for functions belonging to $\mathcal{C}\left(\mathbb{R}^{+}\right)$.

We begin with the following pointwise convergence theorem.

Theorem 1 Let $\psi \in \Psi$ and $\varphi \in \Phi$. Let $f$ be a bounded function. If $x \in \mathbb{R}^{+}$is a continuity point for $f$ then

$$
\lim _{n \rightarrow \infty}\left(S_{n}^{\varphi, \psi} f\right)(x)=f(x), \quad \text { for } \quad x \in \mathbb{R}^{+} .
$$

Proof Let $x \in \mathbb{R}^{+}$be a continuity point of $f$. Since $\varphi \in \Phi$ and $\psi \in \Psi$, we have 


$$
\left|\left(S_{n}^{\varphi, \psi} f\right)(x)-f(x)\right| \leq \sum_{k=-\infty}^{\infty}\left|\varphi\left(e^{-k} x^{n}\right)\right| n \int_{0}^{\infty}\left|\psi\left(e^{-k} u^{n}\right)\right||f(u)-f(x)| \frac{d u}{u}
$$

For a fixed $\varepsilon>0$ by the continuity of $f$ at $x$, there exists $\delta=\delta(\varepsilon)>0$ such that if $|\log u-\log x|=\left|\log \frac{u}{x}\right|<\delta$, then $|f(u)-f(x)|<\varepsilon$. We write

$$
\begin{aligned}
& \left|\left(S_{n}^{\varphi, \psi} f\right)(x)-f(x)\right| \\
& \quad \leq \sum_{k=-\infty}^{\infty}\left|\varphi\left(e^{-k} x^{n}\right)\right| n\left\{\int_{\left|\log \left(\frac{u}{x}\right)\right|<\delta}+\int_{\left|\log \left(\frac{u}{x}\right)\right| \geq \delta}\right\}\left|\psi\left(e^{-k} u^{n}\right)\right||f(u)-f(x)| \frac{d u}{u} \\
& \quad:=I_{1}+I_{2} .
\end{aligned}
$$

Setting $e^{-k} u^{n}=t$ by assumptions ( $\left.\varphi .2\right)$ and ( $\left.\psi .2\right)$

$$
\begin{aligned}
I_{1} & =\sum_{k=-\infty}^{\infty}\left|\varphi\left(e^{-k} x^{n}\right)\right| n \int_{\left|\log \left(\frac{u}{x}\right)\right|<\delta}\left|\psi\left(e^{-k} u^{n}\right)\right||f(u)-f(x)| \frac{d u}{u} \\
& \leq \varepsilon \sum_{k=-\infty}^{\infty}\left|\varphi\left(e^{-k} x^{n}\right)\right| \int_{\left|\log \left(\frac{\left(t^{k}\right)^{\frac{1}{n}}}{x}\right)\right|<\delta}|\psi(t)| \frac{d t}{t} \leq \varepsilon M_{0}(\varphi) \tilde{M}_{0}(\psi) .
\end{aligned}
$$

As to $I_{2}$ we have, by the boundedness of $f$

$$
\begin{aligned}
I_{2} \leq \sum_{k=-\infty}^{\infty}\left|\varphi\left(e^{-k} x^{n}\right)\right| n \int_{\left|\log \left(\frac{u}{x}\right)\right| \geq \delta}\left|\psi\left(e^{-k} u^{n}\right)\right||f(u)-f(x)| \frac{d u}{u} \\
\leq 2\|f\|_{\infty} \sum_{k=-\infty}^{\infty}\left|\varphi\left(e^{-k} x^{n}\right)\right| \int_{0}^{x^{n} e^{-n \delta} e^{-k}}|\psi(t)| \frac{d t}{t} \\
\quad+2\|f\|_{\infty} \sum_{k=-\infty}^{\infty}\left|\varphi\left(e^{-k} x^{n}\right)\right| \int_{x^{n} e^{n \delta} e^{-k}}^{\infty}|\psi(t)| \frac{d t}{t} \\
=I_{2}^{1}+I_{2}^{2} .
\end{aligned}
$$

We have

$$
\begin{aligned}
I_{2}^{1}= & 2\|f\|_{\infty} \sum_{k=-\infty}^{\infty}\left|\varphi\left(e^{-k} x^{n}\right)\right| \int_{0}^{x^{n} e^{-n \delta} e^{-k}}|\psi(t)| \frac{d t}{t} \\
& =2\|f\|_{\infty}\left\{\sum_{\left|\log \left(e^{-k} x^{n}\right)\right|<n \delta / 2}+\sum_{\left|\log \left(e^{-k} x^{n}\right)\right| \geq n \delta / 2}\right\}\left|\varphi\left(e^{-k} x^{n}\right)\right| \int_{0}^{x^{n} e^{-n \delta} e^{-k}}|\psi(t)| \frac{d t}{t} \\
& :=I_{2}^{1,1}+I_{2}^{1,2} .
\end{aligned}
$$

For $I_{2}^{1,1}$ since $\left|\log \left(e^{-k} x^{n}\right)\right|<n \delta / 2$ we have $x^{n} e^{-n \delta} e^{-k}<e^{-n \delta} e^{n \delta / 2}=e^{-n \delta / 2}$ and so for fixed $x, \delta$ we obtain

$$
\int_{0}^{x^{n} e^{-n \delta} e^{-k}}|\psi(t)| \frac{d t}{t} \leq \int_{0}^{e^{-n \delta / 2}}|\psi(t)| \frac{d t}{t}
$$

and for the absolute continuity of the integral of $\psi$ we have that, for large $n$ 


$$
\int_{0}^{x^{n} e^{-n \delta} e^{-k}}|\psi(t)| \frac{d t}{t} \leq \varepsilon
$$

for every $k$ such that $\left|\log \left(e^{-k} x^{n}\right)\right|<n \delta / 2$. As to $I_{2}^{1,2}$

$$
\begin{aligned}
I_{2}^{1,2} & =2\|f\|_{\infty} \sum_{\left|\log \left(e^{-k} x^{n}\right)\right| \geq n \delta / 2}\left|\varphi\left(e^{-k} x^{n}\right)\right| \int_{0}^{x^{n} e^{-n \delta} e^{-k}}|\psi(t)| \frac{d t}{t} \\
& <2\|f\|_{\infty} \widetilde{M}_{0}(\psi) \sum_{\left|\log \left(e^{-k} x^{n}\right)\right| \geq n \delta / 2}\left|\varphi\left(e^{-k} x^{n}\right)\right|
\end{aligned}
$$

and so for large $n$ using property $(\varphi .3)$ we have $I_{2}^{1,2} \leq 2\|f\|_{\infty} \widetilde{M}_{0}(\psi) \varepsilon$. The term $I_{2}^{2}$ is is estimated in a similar way, so the assertion follows.

Using essentially the same reasoning employed in the previous theorem we can prove the following uniform convergence result.

Theorem 2 Let $f \in \mathcal{C}\left(\mathbb{R}^{+}\right) \psi \in \Psi$ and $\varphi \in \Phi$ then

$$
\lim _{n \rightarrow \infty}\left\|\left(S_{n}^{\varphi, \psi} f\right)(x)-f(x)\right\|_{\infty}=0 .
$$

\section{An asymptotic formula}

In this section we will assume that $\varphi \in \Phi$ and $\psi \in \Psi$ are such that their moments $m_{\nu}(\varphi, u)$ and $\tilde{m}_{\nu}(\psi)$ up to the order $r \in \mathbb{N}$ are finite and moreover $m_{\nu}(\varphi, u)=m_{\nu}(\varphi)$ are all independent of $u \in \mathbb{R}^{+}$, for $v=1,2, \ldots r$.

We have the following result

Theorem 3 Let $\varphi \in \Phi, \psi \in \Psi$ be such that $M_{r}(\varphi)$ and $\tilde{M}_{r}(\psi)$ are both finite. Let $f \in L^{\infty}\left(\mathbb{R}^{+}\right)$and let $x \in \mathbb{R}^{+}$be fixed. If for $r \in \mathbb{N}, f \in C^{r}$ locally at the point $x$

$$
\left(S_{n}^{\varphi, \psi} f\right)(x)=\sum_{j=0}^{r} \frac{\left(\Theta^{j} f\right)(x)}{j ! n^{j}} \sum_{\nu=0}^{j}\left(\begin{array}{l}
j \\
v
\end{array}\right) m_{j-\nu}(\varphi) \tilde{m}_{\nu}(\psi)+o\left(n^{-r}\right), \quad n \rightarrow+\infty .
$$

Proof Using Proposition 1 we have

$$
f(u)=f(x)+(\Theta f)(x) \log \left(\frac{u}{x}\right)+\frac{\left(\Theta^{2} f\right)(x)}{2 !} \log ^{2}\left(\frac{u}{x}\right)+\cdots+\frac{\left(\Theta^{r} f\right)(x)}{r !} \log ^{r}\left(\frac{u}{x}\right)+h\left(\frac{u}{x}\right) \log ^{r}\left(\frac{u}{x}\right),
$$

where $h$ is a bounded function and $h(y) \rightarrow 0$ for $y \rightarrow 1$. Thus 


$$
\begin{aligned}
\left(S_{n}^{\varphi, \psi} f\right)(x)-f(x)= & \sum_{k=-\infty}^{\infty} \varphi\left(e^{-k} x^{n}\right) n \int_{0}^{\infty} \psi\left(e^{-k} u^{n}\right)(f(u)-f(x)) \frac{d u}{u} \\
= & \sum_{k=-\infty}^{\infty} \varphi\left(e^{-k} x^{n}\right) n \int_{0}^{\infty} \psi\left(e^{-k} u^{n}\right)(\Theta f)(x) \log \left(\frac{u}{x}\right) \frac{d u}{u} \\
& +\sum_{k=-\infty}^{\infty} \varphi\left(e^{-k} x^{n}\right) n \int_{0}^{\infty} \psi\left(e^{-k} u^{n}\right) \frac{\left(\Theta^{2} f\right)(x)}{2 !} \log ^{2}\left(\frac{u}{x}\right) \frac{d u}{u} \\
& +\cdots+\sum_{k=-\infty}^{\infty} \varphi\left(e^{-k} x^{n}\right) n \int_{0}^{\infty} \psi\left(e^{-k} u^{n}\right) \frac{\left(\Theta^{r} f\right)(x)}{r !} \log ^{r}\left(\frac{u}{x}\right) \frac{d u}{u} \\
& +\sum_{k=-\infty}^{\infty} \varphi\left(e^{-k} x^{n}\right) n \int_{0}^{\infty} \psi\left(e^{-k} u^{n}\right) h\left(\frac{u}{x}\right) \log ^{r}\left(\frac{u}{x}\right) \frac{d u}{u} \\
=: & I_{1}+I_{2}+\cdots+I_{r}+R .
\end{aligned}
$$

For every $j=1,2, \ldots, r$ we have, with the change of variable $e^{-k} u^{n}=t$,

$$
\begin{aligned}
I_{j} & =\sum_{k=-\infty}^{\infty} \varphi\left(e^{-k} x^{n}\right) n \int_{0}^{\infty} \psi\left(e^{-k} u^{n}\right) \frac{\left(\Theta^{j} f\right)(x)}{j !} \log ^{j}\left(\frac{u}{x}\right) \frac{d u}{u} \\
& =\frac{\left(\Theta^{j} f\right)(x)}{j !} \sum_{k=-\infty}^{\infty} \varphi\left(e^{-k} x^{n}\right) \int_{0}^{\infty} \psi(t)\left(\frac{1}{n}(\log t+k)-\log x\right)^{j} \frac{d t}{t} \\
& =\frac{\left(\Theta^{j} f\right)(x)}{j ! n^{j}} \sum_{k=-\infty}^{\infty} \varphi\left(e^{-k} x^{n}\right) \int_{0}^{\infty} \psi(t)\left(\log t+k-\log x^{n}\right)^{j} \frac{d t}{t} \\
& =\frac{\left(\Theta^{j} f\right)(x)}{j ! n^{j}} \sum_{v=0}^{j}\left(\begin{array}{l}
j \\
v
\end{array}\right) \sum_{k=-\infty}^{\infty} \varphi\left(e^{-k} x^{n}\right)\left(k-\log x^{n}\right)^{j-v} \int_{0}^{\infty} \psi(t) \log ^{v} t \frac{d t}{t} \\
& =\frac{\left(\Theta^{j} f\right)(x)}{j ! n^{j}} \sum_{v=0}^{j}\left(\begin{array}{l}
j \\
v
\end{array}\right) m_{j-\nu}(\varphi) \tilde{m}_{\nu}(\psi) .
\end{aligned}
$$

Now we evaluate the term

$$
R=\sum_{k=-\infty}^{\infty} \varphi\left(e^{-k} x^{n}\right) n \int_{0}^{\infty} \psi\left(e^{-k} u^{n}\right) h\left(\frac{u}{x}\right) \log ^{r}\left(\frac{u}{x}\right) \frac{d u}{u} .
$$

Let $\varepsilon>0$ be fixed and let $\delta$ be such that $|h(t)|<\varepsilon$ whenever $|\log t|<\delta$. So we have

$$
\begin{aligned}
|R| & \leq \sum_{k=-\infty}^{\infty}\left|\varphi\left(e^{-k} x^{n}\right)\right| n\left\{\int_{|\log (u / x)|<\delta}+\int_{|\log (u / x)| \geq \delta}\right\}\left|\psi\left(e^{-k} u^{n}\right)\right|\left|h\left(\frac{u}{x}\right)\right|\left|\log ^{r}\left(\frac{u}{x}\right)\right| \frac{d u}{u} \\
& =: R_{1}+R_{2} .
\end{aligned}
$$

For $R_{1}$ we have, by the same change of variable as before, 


$$
\begin{aligned}
R_{1} & \leq \varepsilon \sum_{k=-\infty}^{\infty}\left|\varphi\left(e^{-k} x^{n}\right)\right| n \int_{|\log (u / x)|<\delta}\left|\psi\left(e^{-k} u^{n}\right)\right|\left|\log ^{r} \frac{u}{x}\right| \frac{d u}{u} \\
& =\varepsilon \sum_{k=-\infty}^{\infty}\left|\varphi\left(e^{-k} x^{n}\right)\right| \int_{\left|\log \left(\frac{1^{1 / n} e^{k / n}}{x}\right)\right|<\delta}|\psi(t)|\left|\log ^{r}\left(\frac{t^{1 / n} e^{k / n}}{x}\right)\right| \frac{d t}{t} \\
& =\frac{\varepsilon}{n^{r}} \sum_{k=-\infty}^{\infty}\left|\varphi\left(e^{-k} x^{n}\right)\right| \int_{\left|\log \left(\frac{t^{1 / n_{e} / n}}{x}\right)\right|<\delta}|\psi(t)|\left|\log t+k-\log x^{n}\right|^{r} \frac{d t}{t} .
\end{aligned}
$$

Since $\left|\log t+k-\log x^{n}\right|^{r} \leq 2^{r-1}\left(|\log t|^{r}+\left|k-\log x^{n}\right|^{r}\right)$ we have

$$
R_{1} \leq \frac{\varepsilon 2^{r-1}}{n^{r}}\left(M_{0}(\varphi) \tilde{M}_{r}(\psi)+\widetilde{M}_{0}(\psi) M_{r}(\varphi)\right) .
$$

For $R_{2}$ we have

$$
\begin{aligned}
R_{2} \leq & \frac{\|h\|_{\infty}}{n^{r}} \sum_{k=-\infty}^{\infty}\left|\varphi\left(e^{-k} x^{n}\right)\right| \int_{\left|\log \left(\frac{t^{k}}{x^{n}}\right)^{1 / n}\right| \geq \delta}|\psi(t)|\left|\log t+k-\log x^{n}\right|^{r} \frac{d t}{t} \\
\leq & \frac{2^{r-1}\|h\|_{\infty}}{n^{r}} \sum_{k=-\infty}^{\infty}\left|\varphi\left(e^{-k} x^{n}\right)\right| \int_{0}^{e^{-n \delta} x^{n} e^{-k}}\left|\psi(t) \| k-\log x^{n}\right|^{r} \frac{d t}{t} \\
& +\frac{2^{r-1}\|h\|_{\infty}}{n^{r}} \sum_{k=-\infty}^{\infty}\left|\varphi\left(e^{-k} x^{n}\right)\right| \int_{e^{n \delta} x^{n} e^{-k}}^{\infty}|\psi(t)|\left|k-\log x^{n}\right|^{r} \frac{d t}{t} \\
& +\frac{2^{r-1}\|h\|_{\infty}}{n^{r}} \sum_{k=-\infty}^{\infty}\left|\varphi\left(e^{-k} x^{n}\right)\right| \int_{e^{n \delta} x^{n} e^{-k}}^{\infty}|\psi(t) \| \log t|^{r} \frac{d t}{t} \\
& +\frac{2^{r-1}\|h\|_{\infty}}{n^{r}} \sum_{k=-\infty}^{\infty}\left|\varphi\left(e^{-k} x^{n}\right)\right| \int_{0}^{e^{-n \delta} x^{n} e^{-k}}|\psi(t) \| \log t|^{r} \frac{d t}{t} \\
=: R_{2}^{1} & +R_{2}^{2}+R_{2}^{3}+R_{2}^{4} .
\end{aligned}
$$

We consider only the term $R_{2}^{1}$, since the other terms can be estimated in a similar way. We have

$$
\begin{aligned}
R_{2}^{1}= & \frac{2^{r-1}\|h\|_{\infty}}{n^{r}} \sum_{k=-\infty}^{\infty}\left|\varphi\left(e^{-k} x^{n}\right)\right|\left|k-\log x^{n}\right|^{r} \int_{0}^{e^{-n \delta} x^{n} e^{-k}}|\psi(t)| \frac{d t}{t}=\frac{2^{r-1}\|h\|_{\infty}}{n^{r}} \\
& \cdot\left(\sum_{\left|\log \left(e^{-k} x^{n}\right)\right|<n \delta / 2}+\sum_{\left|\log \left(e^{-k} x^{n}\right)\right| \geq n \delta / 2}\right)\left|\varphi\left(e^{-k} x^{n}\right)\right|\left|k-\log x^{n}\right|^{r} \int_{0}^{e^{-n \delta} x^{n} e^{-k}}|\psi(t)| \frac{d t}{t} \\
= & : R_{2}^{1,1}+R_{2}^{1,2} .
\end{aligned}
$$

For $R_{2}^{1,1}$ since $\left|\log \left(e^{-k} x^{n}\right)\right|<n \delta / 2$ in an analogous way to Theorem 1 we can obtain

$$
\int_{0}^{e^{-n \delta} x^{n} e^{-k}}|\psi(t)| \frac{d t}{t} \leq \varepsilon
$$

and so 


$$
R_{2}^{1,1} \leq \frac{2^{r-1}\|h\|_{\infty}}{n^{r}} \varepsilon M_{r}(\varphi)
$$

For $R_{2}^{1,2}$ by assumption $(\varphi .3)$ and for large $n$ we have

$$
R_{2}^{1,2} \leq \frac{2^{r-1}\|h\|_{\infty}}{n^{r}} \varepsilon M_{0}(\psi) .
$$

Corollary 1 Under the assumptions of Theorem 3 if moreover the functions $\varphi$ and $\psi$ are such that $m_{i}(\varphi)=\widetilde{m}_{i}(\psi)=0$ for $i=1, \ldots, r-1$ then we have

$$
\lim _{n \rightarrow \infty} n^{r}\left[\left(S_{n}^{\varphi, \psi} f\right)(x)-f(x)\right]=\frac{\left(\Theta^{r} f\right)(x)}{r !}\left(m_{r}(\varphi)+\widetilde{m}_{r}(\psi)\right) .
$$

\section{A quantitative estimate of the convergence}

Here we state a quantitative approximation result for functions $f \in \mathcal{C}\left(\mathbb{R}^{+}\right)$in terms of the following modulus of continuity

$$
\omega(f, \delta):=\sup \{|f(u)-f(v)|:|\log u-\log v| \leq \delta\}, \quad \delta>0 .
$$

Note that $\omega$ satisfies all the classical properties of a modulus of continuity. In particular we will employ the following one:

$$
\omega(f, \lambda \delta) \leq(\lambda+1) \omega(f, \delta)
$$

for every $\delta, \lambda>0$.

We have the following quantitative estimate

Theorem 4 Let $\varphi \in \Phi, \psi \in \Psi$ be such that $M_{1}(\varphi)$ and $\widetilde{M}_{1}(\psi)$ are finite. If $f \in \mathcal{C}\left(\mathbb{R}^{+}\right)$, then for every $\delta>0$ we have

$$
\left|\left(S_{n}^{\varphi, \psi} f\right)(x)-f(x)\right| \leq \omega(f, \delta) M_{0}(\varphi) \tilde{M}_{0}(\psi)+\frac{\omega(f, \delta)}{n \delta}\left(M_{0}(\varphi) \tilde{M}_{1}(\psi)+M_{1}(\varphi) \tilde{M}_{0}(\psi)\right) .
$$

Proof We have

$$
\begin{aligned}
\left|\left(S_{n}^{\varphi, \psi} f\right)(x)-f(x)\right| & \leq \sum_{k=-\infty}^{\infty}\left|\varphi\left(e^{-k} x^{n}\right)\right| n \int_{0}^{\infty}\left|\psi\left(e^{-k} u^{n}\right)\right||f(u)-f(x)| \frac{d u}{u} \\
& \leq \sum_{k=-\infty}^{\infty}\left|\varphi\left(e^{-k} x^{n}\right)\right| n \int_{0}^{\infty}\left|\psi\left(e^{-k} u^{n}\right)\right| \omega\left(f,\left|\log \left(\frac{u}{x}\right)\right|\right) \frac{d u}{u}
\end{aligned}
$$

Then for any $\delta>0$, using the properties of the modulus $\omega$, we obtain 


$$
\begin{aligned}
& \left|\left(S_{n}^{\varphi, \psi} f\right)(x)-f(x)\right| \\
& \leq \sum_{k=-\infty}^{\infty}\left|\varphi\left(e^{-k} x^{n}\right)\right| n \int_{0}^{\infty}\left|\psi\left(e^{-k} u^{n}\right)\right|\left(1+\frac{|\log (u / x)|}{\delta}\right) \omega(f, \delta) \frac{d u}{u} \\
& \leq \sum_{k=-\infty}^{\infty}\left|\varphi\left(e^{-k} x^{n}\right)\right| n \int_{0}^{\infty}\left|\psi\left(e^{-k} u^{n}\right)\right| \omega(f, \delta) \frac{d u}{u} \\
& \quad+\sum_{k=-\infty}^{\infty}\left|\varphi\left(e^{-k} x^{n}\right)\right| n \int_{0}^{\infty}\left|\psi\left(e^{-k} u^{n}\right)\right| \frac{|\log (u / x)|}{\delta} \omega(f, \delta) \frac{d u}{u} \\
& \leq \omega(f, \delta) M_{0}(\varphi) \tilde{M}_{0}(\psi) \\
& \quad+\frac{\omega(f, \delta)}{\delta} \sum_{k=-\infty}^{\infty}\left|\varphi\left(e^{-k} x^{n}\right)\right| n \int_{0}^{\infty}\left|\psi\left(e^{-k} u^{n}\right)\right||\log (u / x)| \frac{d u}{u} .
\end{aligned}
$$

Using the change of variable $e^{-k} u^{n}=t$ we have for the last series

$$
\begin{aligned}
& \sum_{k=-\infty}^{\infty}\left|\varphi\left(e^{-k} x^{n}\right)\right| n \int_{0}^{\infty}\left|\psi\left(e^{-k} u^{n}\right)\right||\log (u / x)| \frac{d u}{u} \\
= & \sum_{k=-\infty}^{\infty}\left|\varphi\left(e^{-k} x^{n}\right)\right| \int_{0}^{\infty}|\psi(t)|\left|\log \left(\frac{t e^{k}}{x^{n}}\right)^{1 / n}\right| \frac{d t}{t} \\
\leq & \frac{1}{n} \sum_{k=-\infty}^{\infty}\left|\varphi\left(e^{-k} x^{n}\right)\right| \int_{0}^{\infty}|\psi(t)|\left(|\log t|+\left|k-\log x^{n}\right|\right) \frac{d t}{t} \\
\leq & \frac{1}{n}\left(M_{0}(\varphi) \tilde{M}_{1}(\psi)+M_{1}(\varphi) \widetilde{M}_{0}(\psi)\right),
\end{aligned}
$$

and so the assertion follows.

As a consequence of the previous theorem we have

Corollary 2 Under the assumption of Theorem 4 we obtain

$$
\left|\left(S_{n}^{\varphi, \psi} f\right)(x)-f(x)\right| \leq A \omega\left(f, \frac{1}{n}\right),
$$

with $A$ an absolute constant depending only on $\varphi$ and $\psi$.

Proof It is sufficient to set in Theorem $4 \delta=\frac{1}{n}$ for any fixed $n$. Then the assertion follows with $A=M_{0}(\varphi) \tilde{M}_{0}(\psi)+M_{1}(\varphi) \tilde{M}_{0}(\psi)+M_{0}(\varphi) \tilde{M}_{1}(\psi)$.

\section{Some examples}

In this section we will apply the previous theory to various specific examples. We recall that the Mellin transform of a function $f: \mathbb{R}^{+} \rightarrow \mathbb{R}$ is defined by (see e.g. $[18,28]$ )

$$
[f]_{M}^{\wedge}(s):=\int_{0}^{\infty} x^{s-1} f(x) d x, \quad(s=i t, t \in \mathbb{R}),
$$


whenever the function $f$ is such that

$$
\int_{0}^{\infty}|f(x)| \frac{d x}{x}<\infty
$$

1) We begin with an important class of functions with compact support, which represents the analogue in the Mellin setting of the classical central B-splines. For every fixed $n \in \mathbb{N}$ we define

$B_{n}(x)=\frac{1}{(n-1) !} \sum_{j=0}^{n}(-1)^{j}\left(\begin{array}{l}n \\ j\end{array}\right)\left(\frac{n}{2}+\log x-j\right)_{+}^{n-1} \quad\left(x \in \mathbb{R}^{+}\right)$,

where, for every $r \in \mathbb{R}, r_{+}$denotes the positive part of the number $r$. Let us consider the second order Mellin spline defined by

$$
B_{2}(x):=(1-|\log x|)_{+}= \begin{cases}1-\log x, & 1<x<e \\ 1+\log x, & e^{-1}<x<1 \\ 0, & \text { otherwise }\end{cases}
$$

We put $\varphi(x)=\psi(x)=B_{2}(x)$. As to the moments of $\psi$ we have that

$$
\begin{aligned}
& \tilde{m}_{0}\left(B_{2}\right)=\widetilde{M}_{0}\left(B_{2}\right)=\int_{e^{-1}}^{1}(1+\log x) \frac{d x}{x}+\int_{1}^{e}(1-\log x) \frac{d x}{x}=1 \\
& \tilde{m}_{1}\left(B_{2}\right)=0 \quad \tilde{M}_{1}\left(B_{2}\right)=\frac{1}{3} .
\end{aligned}
$$

For the moments of $\varphi$ since the function $B_{2}$ has compact support, all the absolute moments $M_{j}\left(B_{2}\right)$ are finite. Indeed the series have a finite numbers of non-zero terms. The values of the algebraic moments can be deduced by the Mellin-Poisson summation formula, and we have (see [9]) that only for $j=0,1$ the corresponding algebraic moments are independent of $x$. Moreover

$$
m_{0}\left(B_{2}\right)=\sum_{k=-\infty}^{\infty} B_{2}\left(e^{-k} x\right)=1, \quad m_{1}\left(B_{2}\right)=0 .
$$

In this case the asymptotic formula reduces to

$$
\lim _{n \rightarrow \infty} n\left[\left(S_{n}^{B_{2}, B_{2}} f\right)(x)-f(x)\right]=0 .
$$

2) Let us consider the generalized Mellin-Jackson kernel, which is defined by (see [12])

$$
J_{\gamma, \beta}(x):=d_{\gamma, \beta} \operatorname{sinc}^{2 \beta}\left(\frac{\log x}{2 \gamma \beta \pi}\right),
$$

where $x \in \mathbb{R}^{+}, \beta \in \mathbb{N}, \gamma \geq 1, d_{\gamma, \beta}$ is a normalization constant, i.e.

$$
d_{\gamma, \beta}^{-1}:=\int_{0}^{+\infty} \operatorname{sinc}^{2 \beta}\left(\frac{\log x}{2 \gamma \beta \pi}\right) \frac{d u}{u} .
$$


It is known that $\left[J_{\gamma, \beta}\right]_{M}^{\wedge}(i v)=0$ for $|v| \geq 1 / \gamma$, thus $J_{\gamma, \beta}$ is Mellin band-limited. We put $\varphi=J_{\gamma, \beta}(x)$. Using the Mellin-Poisson summation formula (see [9]) we obtain

$$
\sum_{k=-\infty}^{\infty} J_{\gamma, \beta}\left(e^{k} x\right)=\sum_{k=-\infty}^{\infty}\left[J_{\gamma, \beta}\right]_{M}^{\wedge}(2 k \pi i) x^{-2 k \pi i}=\left[J_{\gamma, \beta}\right]_{M}^{\wedge}(0)=1 .
$$

So we can prove that assumptions $(\varphi .1),(\varphi .2)$ and $(\varphi .3)$ are satisfied, see [9]. Concerning the moments, using again the Mellin-Poisson summation formula for the derivatives as in [9], one has $m_{1}\left(J_{\gamma, \beta}\right)=0$, and, for $\beta>3 / 2$, (see [12])

$$
m_{2}\left(J_{\gamma, \beta}\right)=d_{\gamma, \beta} \int_{0}^{+\infty} \operatorname{sinc}^{2 \beta}\left(\frac{\log x}{2 \gamma \beta \pi}\right) \log ^{2} x \frac{d x}{x}=: A_{\gamma, \beta}<+\infty .
$$

Moreover, $M_{2}\left(J_{\gamma, \beta}\right)<+\infty$. For the function $\psi$ we put $\psi(x)=B_{2}(x)$ the previous spline. We have that

$$
\tilde{m}_{2}\left(B_{2}\right)=\tilde{M}_{2}\left(B_{2}\right)=\frac{1}{6}
$$

Therefore the assumptions of the previous theorems are satisfied, with $r=2$. In particular we obtain the following Voronovskaja formula, for $f \in C\left(\mathbb{R}^{+}\right)$of class $C^{(2)}$ locally at the point $x \in \mathbb{R}^{+}$:

$$
\lim _{n \rightarrow+\infty} n^{2}\left[\left(S_{n}^{J_{\gamma, \beta}, B_{2}} f\right)(x)-f(x)\right]=\frac{\Theta^{2} f(x)}{2}\left(A_{\gamma, \beta}+\frac{1}{6}\right) .
$$

Acknowledgements The authors wish to thank the anonymous referees for their comments which improve the presentation of the paper.

Funding Open access funding provided by Università degli Studi di Perugia within the CRUI-CARE Agreement.

\section{Compliance with ethical standards}

Conflict of interest The authors declare that they have no conflict of interest.

Open Access This article is licensed under a Creative Commons Attribution 4.0 International License, which permits use, sharing, adaptation, distribution and reproduction in any medium or format, as long as you give appropriate credit to the original author(s) and the source, provide a link to the Creative Commons licence, and indicate if changes were made. The images or other third party material in this article are included in the article's Creative Commons licence, unless indicated otherwise in a credit line to the material. If material is not included in the article's Creative Commons licence and your intended use is not permitted by statutory regulation or exceeds the permitted use, you will need to obtain permission directly from the copyright holder. To view a copy of this licence, visit http://creativecommons.org/licenses/by/4.0/.

\section{References}

1. Angeloni, L., Costarelli, D., Vinti, G.: A characterization of the absolute continuity in terms of convergence in variation for the sampling Kantorovich operators. Mediterr. J. Math. 16(2), 44 (2019) 
2. Asdrubali, F., Baldinelli, G., Bianchi, F., Costarelli, D., Rotili, A., Seracini, M., Vinti, G.: Detection of thermal bridges from thermographic images by means of image processing approximation algorithms. Appl. Math. Comput. 317, 160-171 (2018)

3. Balsamo, S., Mantellini, I.: On linear combinations of general exponential sampling series. Results Math. 74, 180 (2019)

4. Bardaro, C., Butzer, P.L., Mantellini, I.: The exponential sampling theorem of signal analysis and the reproducing kernel formula in the Mellin transform setting. Sampl. Theory Signal Image Process. 13(1), 35-66 (2014)

5. Bardaro, C., Butzer, P.L., Mantellini, I., Schmeisser, G.: On the Paley-Wiener theorem in the Mellin transform setting. J. Approx. Theory 207, 60-75 (2016)

6. Bardaro, C., Butzer, P.L., Mantellini, I., Schmeisser, G.: A fresh approach to the Paley-Wiener theorem for Mellin transforms and the Mellin-Hardy spaces. Math. Nachr. 290, 2759-2774 (2017)

7. Bardaro, C., Butzer, P.L., Stens, R.L., Vinti, G.: Kantorovich-type generalized sampling series in the setting of Orlicz spaces. Sampl. Theory Signal Image Process. 6(1), 29-52 (2007)

8. Bardaro, C., Butzer, P.L., Stens, R.L., Vinti, G.: Prediction by samples from the past with error estimates covering discontinuous signals. IEEE Trans. Inf. Theory 56(1), 614-633 (2010)

9. Bardaro, C., Faina, L., Mantellini, I.: A generalization of the exponential sampling series and its approximation properties. Math. Slovaca 67(6), 1481-1496 (2017)

10. Bardaro, C., Faina, L., Mantellini, I.: Quantitative Voronovskaja formulae for generalized Durrmeyer sampling type series. Math. Nachr. 289(14-15), 1702-1720 (2016)

11. Bardaro, C., Mantellini, I.: A note on the Voronovskaja theorem for Mellin-Fejer convolution operators. Appl. Math. Lett. 24, 2064-2067 (2011)

12. Bardaro, C., Mantellini, I.: Asymptotic formulae for linear combinations of generalized sampling type operators. Z. Anal. Anwend. 32, 279-296 (2013)

13. Bardaro, C., Mantellini, I.: Asymptotic expansion of generalized Durrmeyer sampling type series. Jaen J. Approx. 6(2), 143-165 (2014)

14. Bardaro, C., Mantellini, I.: On pointwise approximation properties of multivarate sami-discrete sampling type operators. Results Math. 72, 1449-1472 (2017)

15. Bardaro, C., Mantellini, I., Schmeisser, G.: Exponential sampling series: convergence in Mellin-Lebesgue spaces. Results Math. 74, 119 (2019)

16. Bertero, M., Pike, E.R.: Exponential sampling method for Laplace and other dilationally invariant transforms I. Singular-system analysis. II. Examples in photon correction spectroscopy and Frauenhofer diffraction. Inverse Problems 7, 1-20, 21-41 (1991)

17. Butzer, P.L., Jansche, S.: A direct approach to the Mellin transform. J. Fourier Anal. Appl. 3, 325-375 (1997)

18. Butzer, P.L., Jansche, S.: The exponential sampling theorem of signal analysis. Atti Sem. Mat. Fis. Univ. Modena Suppl. 46, 99-122 (1998). (special issue dedicated to Professor Calogero Vint)

19. Casasent, D. (ed.): Optical Data Processing, pp. 241-282. Springer, Berlin (1978)

20. Costarelli, D., Minotti, A., Vinti, G.: Approximation of discontinuous signals by sampling Kantorovich series. J. Math. Anal. Appl. 450(2), 1083-1103 (2017)

21. Costarelli, D., Sambucini, A.R.: Approximation results in Orlicz spaces for sequences of Kantorovich max-product neural network operators. RM 73, 15 (2018). https://doi.org/10.1007/s00025-018-0799-4

22. Costarelli, D., Sambucini, A.R., Vinti, G.: Convergence in Orlicz spaces by means of the multivariate max-product neural network operators of the Kantorovich type. Neural Comput. Appl. 31(9), 50695078 (2019). https://doi.org/10.1007/s00521-018-03998-6

23. Costarelli, D., Seracini, M., Vinti, G.: A segmentation procedure of the pervious area of the aorta artery from CT images without contrast medium. Math. Methods Appl. Sci. 43(1), 114-133 (2020)

24. Costarelli, D., Vinti, G.: Saturation by the Fourier transform method for the sampling Kantorovich series based on bandlimited kernels. Anal. Math. Phys. 9(4), 2263-2280 (2019)

25. Costarelli, D., Vinti, G.: Inverse results of approximation and the saturation order for the sampling Kantorovich series. J. Approx. Theory 242, 64-82 (2019)

26. Gori, F.: Sampling in optics. In: Marks II, R.J. (ed.) Advances Topics in Shannon Sampling and Interpolation Theory, pp. 37-83. Springer, New York (1993)

27. Higgins, J.R.: Sampling Theory in Fourier and Signal Analysis, Foundations. Oxford University Press, Oxford (1996)

28. Mamedov, R.G.: The Mellin Transform and Approximation Theory. Elm, Baku (1991). (in Russian)

29. Ostrowsky, N., Sornette, D., Parker, P., Pike, E.R.: Exponential sampling method for light scattering polydispersity analysis. Opt. Acta 28, 1059-1070 (1994)

30. Schumaker, L.L.: Spline Functions: Basic Theory. Wiley, New York (1981) 
31. Vinti, G., Zampogni, L.: A unifying approach to convergence of linear sampling type operators in Orlicz spaces. Adv. Differ. Equ. 16(5-6), 573-600 (2011)

Publisher's Note Springer Nature remains neutral with regard to jurisdictional claims in published maps and institutional affiliations. 